Alex SANDRo Rolland SOUZA ${ }^{1}$

Wagner de Menezes Medeiros JUNIOR ${ }^{2}$

Bruno Biller Teixeira Fernandes de Araújo² Isabea Cristina Coutinho de Albuquerque Nenva Coeho3

Gláucia VirGína de Queroz Lins Guerra ${ }^{3}$

Artigo Original

Trabalho de parto induzido/métodos

Cesárea

Nascimento vaginal após cesárea

Parto

Keywords

Labor, induced/methods

Cesarean section

Vaginal birth after cesarean

Parturition

Correspondênci

Alex Sandro Rolland de Souza

Rua dos Coelhos, 300 - Boa Visto

CEP: $50070-550$

Recife (PE), Brasil

Recebido

16/08/2014

Aceito com modificacōoes

$29 / 01 / 2015$

\section{Método mecânico de indução do parto em gestantes de alto risco com cesariana anterior}

\author{
Mechanical method of induction of labor in high-risk \\ pregnant women with previous cesarean section
}

\section{Resumo}

OBJETIVO: Descrever os desfechos materno-fetais com o uso da sonda de Foley para indução do trabalho de parto em gestantes de alto risco com cesariana anterior. MÉTODOS: Foi realizado um estudo de intervenção e descritivo, no período de novembro de 2013 a junho de 2014. Foram incluídas 39 gestantes a termo, com feto vivo, apresentação cefálica, peso estimado $<4.000 \mathrm{~g}$, cesariana prévia, com indicação de indução do trabalho de parto, escore de Bishop $\leq 6$ e índice de líquido amniótico $>5 \mathrm{~cm}$. A sonda de Foley $\mathrm{n}^{\circ} 16 \mathrm{~F}$ foi introduzida, por no máximo 24 horas, sendo considerado satisfatória quando a paciente entrou em trabalho de parto nas primeiras 24 horas. RESULTADOS: $\bigcirc$ trabalho de parto foi induzido satisfatoriamente em 79,5\% das gestantes. Nove mulheres evoluíram para parto vaginal $(23,1 \%)$, com uma frequência de 18\% de partos vaginais ocorridos dentro de 24 horas. As principais indicações da indução do parto foram as síndromes hipertensivas (75\%). As médias dos intervalos entre

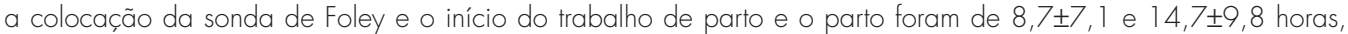
respectivamente. A eliminação de mecônio foi observada em 2 pacientes e o escore de Apgar $<7$ no primeiro minuto foi observado em 5 recém-nascidos (12,8\%). CONCLUSÕES: A sonda de Foley é uma alternativa para indução do trabalho de parto em gestantes com cesariana anterior, apesar da baixa taxa de parto vaginal.

\section{Abstract}

PURPOSE: To describe the maternal and fetal outcomes with the use of the Foley catheter for induction of labor in highrisk pregnant women with previous caesarean section. METHODS: An interventive and descriptive study was conducted from November 2013 to June 2014. A total of 39 pregnant women at term, with a live fetus, cephalic presentation, estimated fetal weight $<4,000 \mathrm{~g}$, with previous cesarean section, medical indications for induction of labor, Bishop score $\leq 6$ and amniotic fluid index $>5 \mathrm{~cm}$ were included. A number $16 \mathrm{~F}$ Foley catheter was introduced for a maximum of 24 hours, and was considered to be satisfactory when the patient began labor within 24 hours. RESULTS: Labor was successfully induced in $79.5 \%$ of pregnant women. Nine women achieved vaginal delivery $(23.1 \%)$, with a frequency of $18 \%$ of vaginal births occurring within 24 hours. The main indications for the induction of labor were hypertensive disorders (75\%). The mean interval between the placement of the Foley catheter and the beginning of labor and delivery

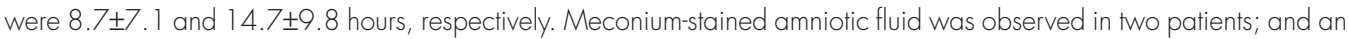
Apgar score $<7$ in the first minute was detected in 5 newborns (12.8\%). CONCLUSIONS: The Foley catheter is an alternative for the induction of labor in women with previous caesarean section, despite the low vaginal delivery rate.
Centro de Atenção à Mulher do Instituto de Medicina Integral Professor Fernando Figueira - IMIP - Recife (PE), Brasil IInstituto de Medicina Integral Professor Fernando Figueira - IMIP; Departamento Materno Infantil, Universidade Federal de Pernambuco UFPE - Recife (PE), Brasil.

${ }^{2}$ Curso Acadêmico de Medicina, Faculdade Pernambucana de Saúde - FPS - Cidade (PE), Brasil.

${ }^{3}$ Enfermaria de Gestação de Alto Risco, Centro de Atenção à Mulher, Instituto de Medicina Integral Professor Fernando Figueira IMIP - Recife (PE), Brasil.

Conflito de interesses: não há. 
Introdução

Durante as duas últimas décadas, os índices de cesarianas se elevaram em muitos países de renda elevada ${ }^{1}$. Nos países de baixa renda, as taxas de cesárea variam bastante. No Brasil, no ano de 2011, a proporção de cesarianas chegou a aproximadamente $54 \%{ }^{2}$.

Existe uma tendência dos obstetras a realizar uma nova cesariana nas gestantes com cicatriz uterina anterior, apesar de não haver respaldo na literatura ${ }^{3,4}$. Um estudo observou que a taxa de parto vaginal em mulheres com cesariana anterior e trabalho de parto espontâneo foi de $72,1 \%$ e que o risco para não ocorrer o parto vaginal está associado a fatores como índice de massa corpórea (IMC) maior do que $25 \mathrm{~kg} / \mathrm{m}^{2}$, idade gestacional maior do que a $40^{\mathrm{a}}$ semana e dilatação cervical menor do que $4 \mathrm{~cm}$ na admissão 5 .

Em duas revisões sistemáticas os autores concluíram que a realização de uma cesariana eletiva ou a indução do parto ou o trabalho de parto espontâneo em mulheres com uma cesárea anterior podem estar associados a benefícios (parto vaginal) e malefícios (possibilidade de ruptura uterina). Porém, não há evidência suficiente baseada em ensaios clínicos para se determinar qual é a melhor conduta ${ }^{6,7}$.

Nesse cenário, o principal risco das mulheres com cesariana prévia é a ruptura uterina, principalmente quando o parto é induzido ${ }^{8}$. Assim, estudos foram realizados para fundamentar decisões clínicas sobre os métodos de indução do parto com menores taxas de complicações?. Porém, ainda não existem informações suficientes baseadas em ensaios clínicos para decidir qual método é preferível ${ }^{9}$.

Outra revisão sistemática, de estudos de coorte retrospectiva em mulheres com cesariana anterior, comparou a indução do parto com o parto espontâneo. Foi observado que a indução causa menor taxa de parto vaginal e maiores taxas de ruptura uterina/deiscência de cicatriz uterina e hemorragia pós-parto. Entretanto, destaca-se que dos oito estudos incluídos apenas dois utilizaram a sonda de Foley ${ }^{10}$.

A sonda de Foley é usada na indução do trabalho de parto, principalmente nos casos em que há contraindicação para uso das prostaglandinas, como na presença de cesariana anterior ${ }^{8,11,12}$. Esse método consiste na inserção da sonda ultrapassando o orifício interno do colo uterino, enchimento do balão e tração por fixação da sonda à perna da gestante. O mecanismo de ação ocorre pela ação mecânica e por liberação de prostaglandinas devido à separação do córion da decídua ${ }^{12,13}$.

Estudos e revisões da literatura sugerem que o uso da sonda de Foley é efetivo para indução do parto, cursando com menor frequência de hiperestimulação, taquissistolia e ruptura uterina quando comparado às prostaglandinas ${ }^{11-13}$. Porém, sugere-se um aumento na frequência de infecção materna e fetal ${ }^{14}$, devendo ser respeitadas as contraindicações, sobretudo no caso de placenta de inserção baixa ${ }^{12,15}$.
Um estudo de coorte retrospectiva foi realizado na França com 151 mulheres para avaliar a segurança e eficácia da indução do parto em mulheres com cesárea anterior, que usaram o cateter de Foley para amadurecimento cervical. A taxa de parto vaginal foi de $54 \%$ e houve ruptura uterina em 2 pacientes ${ }^{16}$. Outro estudo retrospectivo no mesmo país com 135 pacientes relatou que o amadurecimento do colo do útero com sonda de Foley é uma boa opção em pacientes com cicatriz uterina prévia, ocorrendo o desfecho de parto vaginal em $64,1 \%$ das mulheres, sem nenhuma complicação materna ou fetal ${ }^{17}$.

Dessa forma, esse estudo prospectivo, diferente da maioria dos encontrados na literatura, foi realizado com o intuito de descrever os desfechos materno-fetais com o uso da sonda de Foley para induzir o trabalho de parto de feto vivo a termo em gestantes com cesariana anterior.

\section{Métodos}

Foi realizado um estudo de intervenção envolvendo 39 gestantes com cesariana prévia e indicação de indução do trabalho de parto, internadas na enfermaria de gestação de alto risco e no pré-parto do Instituto de Medicina Integral Professor Fernando Figueira (IMIP) no período de novembro de 2013 a junho de 2014.

O IMIP encontra-se situado em Recife (PE), no Nordeste do Brasil. É uma entidade filantrópica (sem fins lucrativos) que atende às comunidades carentes do estado e regiões próximas. É um hospital terciário, credenciado como hospital de ensino pelos Ministérios da Educação e da Saúde e como Centro de Referência Nacional e Estadual na área Materno-Infantil pelo Ministério da Saúde e Sistema Único de Saúde (SUS)-PE, respectivamente. Na Maternidade do IMIP são assistidos anualmente cerca de 6 mil partos, com um elevado percentual de gestações de alto risco (em torno de $50 \%$ ).

O estudo foi submetido ao Comitê de Ética de Pesquisa (CEP) em Seres Humanos (nº 1.911 de 10/11/2010) e, somente após a sua aprovação, teve a coleta de dados iniciada. Todas as pacientes foram devidamente informadas sobre os objetivos e a importância da pesquisa e somente foram incluídas após concordarem livremente em participar do estudo e assinarem o Termo de Consentimento Livre e Esclarecido.

Os critérios de inclusão estabelecidos foram gestação a termo (acima da $37^{a}$ semana), feto vivo, apresentação cefálica de vértice, peso fetal estimado pela ultrassonografia $<4.000 \mathrm{~g}$, índice de liquido amniótico (ILA) $>5 \mathrm{~cm}$, índice de Bishop $\leq 5$ e cesárea em gestação anterior. Foram excluídas as gestantes com outras cicatrizes uterinas prévias que não cesariana segmentar, prova de vitalidade fetal alterada, anomalias fetais, restrição de crescimento fetal, gestação múltipla, sangramento genital e placenta de inserção baixa, corioamnionite. 
A paciente com indicação de indução de trabalho de parto era identificada por médico plantonista e/ou residente da triagem obstétrica e/ou médico assistente da enfermaria de gestação de alto risco da instituição. A paciente recebeu esclarecimento sobre os objetivos e as possíveis consequências da sua participação.

A idade gestacional foi calculada a partir da data da última menstruação (DUM), desde que conhecida e confiável e confirmada pelo primeiro exame ecográfico. Quando a DUM era desconhecida ou havia discordância com o exame ultrassonográfico, a datação da gestação foi realizada com base na primeira ultrassonografia. Antes da indução do parto com a sonda de Foley, todas as gestantes foram submetidas a uma ultrassonografia obstétrica, registrando-se a estimativa do peso do feto, valor do ILA, estática fetal, além da avaliação da vitalidade fetal. Também era realizada avaliação digital do colo uterino, para avaliar a dilatação, esvaecimento, posição, consistência e altura da apresentação, atribuindo a cada um valores zero a três pontos, para obtenção do índice de Bishop ${ }^{18}$.

As gestantes foram acompanhadas no centro obstétrico, onde foi colocada a sonda de Foley por médico capacitado. O colo uterino foi visualizado utilizando um espéculo vaginal e a sonda de Foley n ${ }^{\circ} 16 \mathrm{~F}$ (Solidor, China) foi introduzida dentro do colo uterino ultrapassando o orifício cervical interno e insuflado o balão com $30 \mathrm{~mL}$ de água destilada, além de fixação na face lateral da coxa, aplicando uma tração para baixo ${ }^{18,19}$. A sonda era mantida tracionada por 24 horas ou até sua expulsão, mesmo que o trabalho de parto tivesse iniciado. Caso o parto não fosse deflagrado nesse período, a sonda era retirada e a cesariana indicada por falha de indução.

A paciente ficou internada no pré-parto com livre deambulação e dieta livre, sendo monitorada a dinâmica uterina e a frequência cardíaca fetal a cada 2 horas, enquanto não desencadeava o trabalho de parto, e a cada 15 minutos, a partir do momento que o trabalho de parto foi deflagrado. Todo o trabalho de parto foi acompanhado e conduzido de acordo com a rotina do serviço, pelos médicos plantonistas.

Como características da amostra foram analisadas as seguintes variáveis maternas: idade materna (em anos), idade gestacional (em semanas), número de gestações, paridade, as indicações da indução do parto, o escore de Bishop modificado e o peso fetal estimado pela ultrassonografia. Os desfechos do estudo foram a frequência da indução do parto satisfatória, do parto vaginal e do parto vaginal dentro de 24 horas. A indução do parto foi considerada satisfatória quando a paciente entrou em trabalho de parto nas primeiras 24 horas de indução independente da via de parto ocorrida.

Outras variáveis avaliadas foram: ocorrência de síndrome de hiperestimulação uterina; de cesariana (intraparto e sem trabalho de parto) e suas indicações; a morbidade neonatal grave (convulsões e asfixia neonatal) ou morte perinatal; morbidade materna grave (ruptura uterina, septicemia e admissão na unidade de terapia intensiva - UTI) ou morte materna; modificações da cérvice uterina com 12 e 24 horas; necessidade de ocitocina; tempo transcorrido entre a colocação da sonda e o início do trabalho de parto e o parto; falha de indução; taquissistolia; hipertonia/hipersistolia; ruptura uterina; corioamnionite; necessidade de analgesia peridural; parto vaginal instrumental; e morte materna.

Após o parto, foram avaliadas ainda as complicações neonatais, como mecônio no liquido amniótico, escores de Apgar $<7$ no primeiro e quinto minutos, admissão em UTI neonatal, encefalopatia neonatal e morte perinatal.

As alterações da contratilidade uterina foram classificadas como taquissistolia (presença de 6 ou mais contrações por dois períodos consecutivos de 10 minutos), hipertonia/hipersistolia (contração uterina com duração de 2 minutos ou mais) e a síndrome de hiperestimulação uterina com a presença de alteração da contratilidade uterina (taquissistolia ou hipertonia/hipersistolia) associada à frequência cardíaca fetal (FCF) não tranquilizadora ${ }^{18}$.

A FCF não tranquilizadora foi definida como uma persistência dos batimentos cardíacos $<110$ batimentos por minuto (bpm) ou acima de $160 \mathrm{bpm}$ ou ainda na presença de desacelerações intraparto por meio da auscultação fetal intermitente $e^{18}$. Foi considerada falha de indução a ausência de contrações uterinas eficazes após 24 horas da colocação da sonda de Foley e a distócia de progressão, quando não ocorreu adequada evolução da dilatação cervical, com o trabalho de parto deflagrado, porém ultrapassando a linha de ação do partograma.

Depois da sonda de Foley expulsa devido à dilatação cervical, havendo necessidade, a ocitocina foi iniciada com uma infusão de $2 \mathrm{mIU} / \mathrm{min}$ dobrando esse fluxo a cada $30 \mathrm{~min}$ com infusão máxima de $32 \mathrm{mIU} / \mathrm{min}^{18,19}$.

O trabalho de parto foi definido quando a paciente se encontrava com um mínimo de 3 contrações uterinas em 10 minutos, de no mínino 40 segundos de duração, as quais levassem a modificações da cérvice uterina.

Os dados foram digitados no banco de dados criado no programa Epi Info ${ }^{\mathrm{TM}}$ versão 7.1 para Windows (Atlanta, GA, EUA). Para as variáveis categóricas foram obtidas tabelas de distribuição de frequência, enquanto para as variáveis numéricas foram calculadas medidas de tendência central e de dispersão.

\section{Resultados}

Foram incluídas 39 gestantes. A média da idade materna foi de 29 anos e as medianas dos números de gestações, paridade e do índice de Bishop foram de 2, 1 e 1 , respectivamente. As médias de idade gestacional e do peso fetal estimado pela ultrassonografia foram de 38,3 semanas e $3.197 \mathrm{~g}$, respectivamente (Tabela 1). 
As principais indicações da indução do parto foram síndromes hipertensivas $(75 \% ; \mathrm{n}=30)$, seguida de diabetes $(20 \%, \mathrm{n}=8)$, ruptura prematura das membranas $(5 \% ; \mathrm{n}=2)$, gestação prolongada $(2,5 \% ; \mathrm{n}=1)$ e líquido amniótico diminuído $(2,5 \% ; \mathrm{n}=1)$.

A média do intervalo de tempo entre a colocação da sonda e o início do trabalho de parto foi de 8,7 horas, variando de 1 a 28 horas ( $n=31$; desses apenas 1 mulher foi do grupo de cesariana); entre a colocação da sonda e o parto foi de 14,7 horas, variando de 4 a 36 horas; e da duração do trabalho de parto foi de 7,2 horas ( $\mathrm{n}=9$; todas do grupo de parto vaginal). Houve modificação do colo uterino dentro de 12 horas em $71,8 \%$ das pacientes e com 24 horas em 79,5\%. O uso de ocitocina foi necessário em $84,6 \%$ das gestantes. A indução foi considerada satisfatória em $79,5 \%$ das mulheres, porém 9 evoluíram para parto vaginal e 30 para cesariana, com uma frequência de 17,9\% de partos vaginais ocorridos dentro de 24 horas. Quatro mulheres mantiveram picos hipertensivos após o parto e foram admitidas na UTI obstétrica para controle pressórico, configurando a morbidade materna grave (Tabela 2).

Tabela 1. Características das gestantes com cesariana anterior submetidas à indução do parto com sonda de Foley

\begin{tabular}{lcc}
\hline Característica (n=39) & Valores & Variaccão \\
\hline Idade materna, anos (média \pm DP) & $29 \pm 7,1$ & $17-42$ \\
Gestações anteriores (mediana; IIQ) & $2 ; 2-3$ & $2-7$ \\
Paridade (mediana; IIQ) & $1 ; 1-1$ & $1-4$ \\
Idade gestacional, semanas (média \pm DP) & $38,3 \pm 1,2$ & $37-41,6$ \\
Índice de Bishop (mediana; IIQ) & $1 ; 0-3$ & $0-5$ \\
Peso fetal estimado pela USG & $3.197 \pm 501$ & $1.900-3.908$ \\
\hline
\end{tabular}

DP: desvio padrão; II: intervalo interquartil; USG: ultrassonografia.

Tabela 2. Desfechos maternos das gestantes com cesariana anterior submetida à indução do parto com sonda de Foley

\begin{tabular}{|c|c|}
\hline Desfechos ( $n=39)$ & Valores \\
\hline $\begin{array}{l}\text { Intervalo da colocação da sonda e início do TP } \\
\text { (variação) (n=31) }\end{array}$ & $1-28$ horas \\
\hline $\begin{array}{l}\text { Intervalo da colocacão da sonda e início do TP } \\
\text { (média } \pm \text { DP) ( } n=31 \text { ) }\end{array}$ & $8,7 \pm 7,1$ horas \\
\hline Intervalo da colocação da sonda e o parto (variação) (n=9) & $4-36$ horas \\
\hline Intervalo da colocação da sonda e o parto (média \pm DP) $(n=9)$ & $14,7 \pm 9,8$ horas \\
\hline Modificações da cérvice uterina com 12 horas, n (\%) & $28(71,8)$ \\
\hline Modificações da cérvice uterina com 24 horas, n (\%) & $31(79,5)$ \\
\hline Necessidade de ocitocina, n (\%) & $33(84,6)$ \\
\hline Indução do parto satisfatória, n (\%) & $31(79,5)$ \\
\hline Parto vaginal, n (\%) & $9(23,1)$ \\
\hline Parto vaginal dentro de 24 horas, n (\%) & $7(17,9)$ \\
\hline Cesariana, n (\%) & $30(76,9)$ \\
\hline Cesariana intraparto, n (\%) & $22(56,4)$ \\
\hline Cesariana sem trabalho de parto, n (\%) & $8(20,5)$ \\
\hline Necessidade de internamento da UTI obstétrica, n (\%) & $4(10,3)$ \\
\hline
\end{tabular}

TP: Trabalho de parto; DP: desvio padrão; UTI: unidade de terapia intensiva
Nenhuma gestante apresentou síndrome de hiperestimulação uterina, hipertonia/hipersistolia, taquissistolia, ruptura uterina, corioamnionite, uso de analgesia peridural, parto instrumental ou morte materna.

As indicações de cesariana foram Bishop desfavorável $(n=12)$, distócia de progressão $(n=7)$, picos pressóricos $(n=6)$, frequência cardíaca fetal não tranquilizadora $(n=3)$, desproporção cefalopélvica $(n=2)$ e falha de indução, suspeita de descolamento prematuro da placenta (DPP), distensão segmentar e macrossomia (todos com 1 caso cada).

A análise dos desfechos neonatais mostrou que 5 recém-nascidos (12,8\%) obtiveram escores de Apgar $<7$ no primeiro minuto. Dos cinco, dois precisaram de medidas de reanimação na sala de parto, excluindo o diagnóstico de anóxia no período neonatal imediato. A eliminação de mecônio aconteceu em 2 casos $(5,1 \%)$. Nenhum recém-nascido foi internado em UTI neonatal, nem apresentou encefalopatia, infecção ou morte perinatal.

\section{Discussão}

O uso da sonda de Foley na indução de parto vem sendo estudado, pois se torna uma boa opção quando a paciente apresenta alguma contraindicação ao uso de métodos farmacológicos, como uma cicatriz uterina prévia ${ }^{10-12,16,17}$. A ideia desta pesquisa partiu da necessidade de descrever na nossa população a efetividade e segurança da utilização da sonda na indução do parto e comparar os desfechos com a literatura. No presente estudo foi observada uma taxa de indução satisfatória em aproximadamente $80 \%$ das mulheres. Entretanto, a taxa de parto vaginal foi de $23,1 \%$, sendo que $17,9 \%$ das mulheres pariram dentro das primeiras 24 horas e a taxa de cesariana foi de $77 \%$. Destaca-se que tivemos uma taxa de indução satisfatória adequada, apesar da baixa frequência de parto vaginal, o que pode ter ocorrido devido às características de nossa amostra - gestações de alto risco.

A taxa de parto vaginal foi menor do que a encontrada em outros estudos que utilizaram a sonda de Foley em mulheres com cesariana prévia, o qual variou entre 43,7 e $64,1 \%{ }^{16,17,20,21}$. A alta incidência de cesáreas pode ter ocorrido em razão da nossa pequena amostra, bem como pela tendência de alguns plantonistas em realizar uma nova cesariana nas mulheres com cesariana prévia e também porque nossa principal indicação de indução do trabalho de parto foram as síndromes hipertensivas, que cursaram com seis pacientes submetidas à cesariana por picos hipertensivos.

O que chama a atenção em relação à tendência a essa prática é a elevada quantidade de cesarianas indicadas por Bishop desfavorável e distócia de progressão, situações essas que necessitam de uma fase ativa do trabalho de parto mais prolongado para um diagnóstico mais preciso. 
Além disso, é importante ressaltar que em oito pacientes o trabalho de parto nem sequer foi deflagrado. Essa necessidade resolutiva dos plantonistas corrobora um estudo que sugere que a presença da sonda de Foley é um fator de risco isolado para uma nova cesariana ${ }^{20}$.

Outras publicações observaram que pacientes com cesárea anterior que desencadearam o trabalho de parto espontâneo tiveram menores taxas de cesarianas, quando comparadas com mulheres que iniciaram a indução com sonda de Foley ${ }^{10,20,22}$. Destacamos que todas as nossas gestantes tiveram o trabalho de parto induzido com sonda de Foley, diferente dos estudos mencionados, que compararam a indução com o parto espontâneo.

Outro fator que pode estar envolvido na pequena taxa de parto vaginal observada neste estudo é a ausência de pelo menos um parto vaginal prévio, além da cesárea anterior, na maioria das pacientes envolvidas ${ }^{4,20}$. Esse dado pode ser identificado pelas características maternas do presente estudo, pois a mediana de gestações anteriores das pacientes foi de dois, e a da paridade foi de um.

Ainda sobre efetividade, apesar da reduzida frequência de parto vaginal dentro de 24 horas, consideramos que a sonda de Foley para indução do trabalho de parto foi efetiva devido a aproximadamente $80 \%$ das induções do trabalho de parto terem ocorrido de forma satisfatória. Outros estudos, avaliando o misoprostol, encontraram uma proporção de indução do parto satisfatória oscilando entre 90 e $96 \%{ }^{23,24}$. Talvez a nossa proporção de indução satisfatória não foi tão efetiva quanto esses estudos ${ }^{23,24}$ devido a diferentes métodos de indução utilizados por eles.

Apenas uma paciente apresentou o diagnóstico de falha de indução. Abaixo da porcentagem encontrada em outro estudo, que foi de $12 \%{ }^{17}$. Ressaltamos a dificuldade em definir falha de indução, pois até o momento ainda não se tem um consenso na literatura, dificultando o confronto com outros estudos. Optou-se, então, por usar a definição de ausência de contrações eficazes após 24 horas da introdução da sonda de Foley.

Observou-se ainda no presente estudo que a média do intervalo entre a colocação da sonda e o parto foi inferior a outros descritos na literatura ${ }^{25,26}$. Enquanto no nosso estudo foi encontrado um intervalo médio de 14,7 horas até o parto, outros autores observaram um intervalo médio que variou de 16,5 a 29,6 horas ${ }^{25-27}$. Da mesma forma, a duração do trabalho de parto no presente estudo foi de 7,2 horas, menor do que a média encontrada em outro estudo, que foi de 14,2 horas com a sonda e de 12 horas na indução com misoprostol ${ }^{28}$. Provavelmente, esse tempo médio inferior foi devido à realização de cesarianas, como explicado anteriormente, pela necessidade resolutiva dos médicos plantonistas.

Observamos que a principal indicação de indução foi por síndromes hipertensivas gestacionais (75\%). Divergente da literatura, na qual predomina gestação avançada como principal indicação ${ }^{18,19}$. É importante citar que a instituição onde a pesquisa foi realizada é um centro de gestação de alto risco, favorecendo a presença de pacientes que se enquadram nesse perfil.

Quanto à segurança, estudos sugerem que o uso da sonda de Foley para indução do trabalho de parto pode aumentar de forma geral o risco de corioamnionite ${ }^{14}$. Porém, no presente estudo não foi observado nenhum caso, mesmo nas pacientes incluídas com ruptura prematura das membranas. Destaca-se o pequeno número da amostra e que nossa avaliação foi possível apenas para detecção da corioamnionite clínica.

Especificamente nas pacientes com cesariana prévia existe ainda um risco teórico de ruptura uterina, devido à presença da cicatriz uterina ${ }^{18,19}$. Porém, autores demonstraram que pacientes com cesárea prévia, quando submetidas a uma segunda cirurgia, apresentam maior morbimortalidade materno/fetal (hemorragia, ruptura uterina, asfixia e morte perinatal)em comparação àquelas que evoluíram para parto vaginal ${ }^{29}$. Outro estudo também observou que a sonda de Foley se mostrou superior, pelo menor risco de ruptura, que acontece em aproximadamente $1 \%$ dos $\operatorname{casos}^{8}$. No nosso estudo também foi observado nenhum caso de morbidade por hemorragia ou ruptura uterina, porém, destacamos o pequeno número amostral.

Nas pacientes com cesariana prévia, autores sugerem que os métodos de indução não aumentam outros desfechos desfavoráveis maternos e nem fetais ${ }^{30}$. Em relação às alterações da contratilidade uterina, a sonda de Foley apresentou índices mais baixos de taquissistolia, hipertonia e/ou síndrome de hiperestimulação uterina em comparação às prostaglandinas, segundo revisão sistemática disponibilizada na biblioteca Cochrane $^{13}$. Em nosso estudo não observamos nenhuma das alterações citadas nas pacientes avaliadas.

Quanto às repercussões perinatais, observa-se que não houve nenhuma admissão em UTI neonatal, apesar de dois recém-nascidos apresentarem casos de asfixia neonatal, que foram revertidos com ventilação por pressão positiva, sem ocorrência de morte fetal ou neonatal. É importante ressaltar a nossa pequena amostra.

Como limitações do presente estudo, destacamos que não houve um grupo controle, o tamanho amostral foi pequeno, não houve randomização e o estudo foi descritivo. Assim, sugerimos que novos estudos sejam realizados com um tamanho amostral maior e a presença de um grupo controle, comparando diferentes métodos de indução e em grupos específicos de pacientes, como por exemplo, gestantes com ruptura prematura das membranas e cesariana anterior.

Assim, concluímos que a sonda de Foley é uma alternativa para indução do trabalho de parto em gestantes com cesariana anterior, porém novos estudos devem ser realizados para que essa hipótese seja confirmada. 
1. MacDorman MF, Menacker F, Declercq E. Cesarean birth in the United States: epidemiology, trends, and outcomes. Clin Perinatol. 2008;35(2):293-307.

2. Brasil. Ministério da Saúde. Rede Interagencial de Informações para a Saúde [Internet]. Indicadores de cobertura: proporção de partos cesáreos. 2012 [citado 2014 Mar 10]. Disponível em: <http://tabnet.datasus.gov.br/cgi/tabcgi.exe?idb2012/ f08.def>

3. Eden KB, McDonagh M, Denman MA, Marshall N, Emeis C, Fu $R$, et al. New insights on vaginal birth after cesarean: can it be predicted? Obstet Gynecol. 2010;1 16(4):967-81.

4. Guise JM, Denman MA, Emeis C, Marshall N, Walker M, Fu R, et al. Vaginal birth after cesarean: new insights on maternal and neonatal outcomes. Obstet Gynecol. 2010;1 15(6):1267-78.

5. Siddiqui SA. Obstetric factors for unsuccessful trial of labor in second-order birth following previous cesarean. Ann Saudi Med. 2013;33(4):356-62.

6. Dodd JM, Crowther CA, Grivell RM, Deussen AR. Elective repeat caesarean section versus induction of labour for women with a previous caesarean birth. Cochrane Database Syst Rev. 2014;12:CD004906.

7. Dodd JM, Crowther CA, Huertas E, Guise JM, Horey D. Planned elective repeat caesarean section versus planned vaginal birth for women with a previous caesarean birth. Cochrane Database Syst Rev. 2013;12:CD004224.

8. Bujold E, Blackwell SC, Gauthier RJ. Cervical ripening with transcervical foley catheter and the risk of uterine rupture. Obstet Gynecol. 2004;103(1):18-23.

9. Jozwiak M, Dodd JM. Methods of term labour induction for women with a previous caesarean section. Cochrane Database Syst Rev. 2013;3:CD009792.

10. Rossi AC, Prefumo F. Pregnancy outcomes of induced labor in women with previous cesarean section: a systematic review and meta-analysis. Arch Gynecol Obstet. 2015;291 (2):273-80.

11. Souza AS, Costa AA, Coutinho I, Noronha Neto C, Amorim MM. [Critical analysis of non-pharmacological methods for induction of labor]. Femina. 2010;38(4): 195-201. Portuguese.

12. Surita FG, Cecatti JG, Kruppa F, Tedesco RP, Parpinelli MA. Cervical ripening methods for labor induction. Rev Bras Saúde Mater Infant. 2004;4(2):125-33.

13. Jozwiak M, Bloemenkamp KW, Kelly AJ, Mol BW, Irion O, Boulvain M. Mechanical methods for induction of labour. Cochrane Database Syst Rev. 2012;3:CD001233.

14. Heinemann J, Gillen G, Sanchez-Ramos L, Kaunitz AM. Do mechanical methods of cervical ripening increase infectious morbidity? A systematic review. Am J Obstet Gynecol. 2008; 199(2): 177-87.

15. ACOG Committee on Practice Bulletins - Obstetrics. ACOG Practice Bulletin No. 107: induction of labor. Obstet Gynecol. 2009; 114(2 Pt 1):386-97.
16. Sarreau M, Leufflen L, Monceau E, Tariel D, Villemonteix $P$, Morel $O$, et al. [Balloon catheter for cervical ripening on scarred uterus with unfavorable cervix: multicenter retrospective study of 151 patients]. J Gynecol Obstet Biol Reprod (Paris). 2014;43(1):46-55. French.

17. Rossard L, Arlicot C, Blasco H, Potin J, Denis C, Mercier D, et al. [Cervical ripening with balloon catheter for scared uterus: a threeyear retrospective study]. J Gynecol Obstet Biol Reprod (Paris). 2013;42(5):480-7. French.

18. Royal College of Obstetricians and Gynaecologists; RCOG Clinical Effectiveness Support Unit. Induction of labour. Evidence-based clinical guideline No. 9. London: RCOG Press; 2001.

19. World Health Organization. WHO recommendations for induction of labour. Geneva: World Health Organization; 2011.

20. Sananès N, Rodriguez M, Stora C, Pinton A, Fritz G, Gaudineau $A$, et al. Efficacy and safety of labour induction in patients with a single previous caesarean section: a proposal for a clinical protocol. Arch Gynecol Obstet. 2014;290(4):669-76.

21. Jozwiak $M$, van de Lest $H A$, Burger NB, Dijksterhuis $M G, D e$ Leeuw JW. Cervical ripening with Foley catheter for induction of labor after cesarean section: a cohort study. Acta Obstet Gynecol Scand. 2014;93(3):296-301.

22. Al-Shaikh G, Al-Mandeel H. The outcomes of trial of labour after cesarean section following induction of labour compared to spontaneous labour. Arch Gynecol Obstet. 2013;287(6): 1099-103.

23. Gattás DS, Souza AS, Souza CG, Florentino AV, Nóbrega BV, Fook VP, et al. [Low dose of sublingual misoprostol $(12.5 \mu \mathrm{g})$ for labor induction]. Rev Bras Ginecol Obstet. 2012;34(4): 164-9. Portuguese.

24. Souza AS, Scavuzzi A, Rodrigues DC, Oliveira RD, Feitosa FE, Amorim MM. [Titrated oral solution of misoprostol for labour induction: a pilot study]. Rev Bras Ginecol Obstet. 2010;32(5):208-13. Portuguese.

25. Jozwiak M, Oude Rengerink K, Benthem M, van Beek E, Dijksterhuis $M G$, de Graaf IM, et al. Foley catheter versus vaginal prostaglandin E2 gel for induction of labour at term (PROBAAT trial): an open-label, randomised controlled trial. Lancet. 2011;378(9809):2095-103.

26. Karjane NW, Brock EL, Walsh SW. Induction of labor using a foley balloon, with and without extra-amniotic saline infusion. Obstet Gynecol. 2006;107(2 Pt 1):234-9.

27. Oliveira MV, Oberst PV, Leite GK, Aguemi A, Kenj G, Leme $V D$, et al. [Cervical Foley catheter versus vaginal misoprostol for cervical ripening and induction of labor: a randomized clinical trial]. Rev Bras Ginecol Obstet. 2010;32(7):346-51. Portuguese.

28. TuuliMG, KeeganMB, Odibo AO, RoehIK, Macones GA, CahillAG. Progress of labor in women induced with misoprostol versus the Foley catheter. Am J Obstet Gynecol. 2013;209(3):237.e1-7.

29. Oliveira TA, Aquino MM, Mariani Neto C. Indução do parto em pacientes com cesárea anterior. Femina. 2009;37(8):427-32.

30. Ashwal E, Hiersch L, Melamed N, Ben-Zion M, Brezovsky A, Wiznitzer A, et al. Pregnancy outcome after induction of labor in women with previous cesarean section. J Matern Fetal Neonatal Med. 2014:1-6. [Epub ahead of print] 